\title{
Erratum
}

\section{Erratum to Evidence for the Detection of Non-Endotoxin Pyrogens by the Whole Blood Monocyte Activation Test}

Nina Hasiwa ${ }^{1,2}$, Mardas Daneshian ${ }^{1}$, Peter Bruegger ${ }^{4}$, Stefan Fennrich ${ }^{5}$, Astrid Hochadel ${ }^{2}$, Sebastian Hoffmann ${ }^{6}$, Felix E. Rivera-Mariani ${ }^{3}$, Christoph Rockel ${ }^{7}$, Stefanie Schindler ${ }^{8}$, Ingo Spreitzer ${ }^{9}$, Sandra Stoppelkamp ${ }^{5}$, Kranthi Vysyaraju ${ }^{3}$, and Thomas Hartung ${ }^{1,3}$

${ }^{1}$ CAAT-Europe, University of Konstanz, Konstanz, Germany; ${ }^{2}$ AtaX-Advice, Konstanz, Germany; ${ }^{3}$ Johns Hopkins University, Bloomberg School of Public Health, CAAT, Baltimore, USA; ${ }^{4}$ Novartis, Basel, Switzerland; ${ }^{5}$ Department of Thoracic, Cardiac and Vascular Surgery, Tübingen University Hospital, Tübingen, Germany; ${ }^{6}$ seh consulting + services, Paderborn, Germany; ${ }^{7}$ Enzler Hygiene AG, Center for Hygiene, Pratteln, Switzerland; ${ }^{8}$ Animalfree Research, Zürich, Switzerland; ${ }^{9}$ Paul-Ehrlich Institut (PEI), Langen, Germany

In this $\mathrm{t}^{4}$ report which appeared in ALTEX (2013), 30(2), 169-208, PMID: 23665806

there were errors in Table 14.

Tab. 14 (revised)
Tab. 14: Result of LAL-testing of the 10 spiked drugs used in the MAT validation study

\begin{tabular}{|c|c|c|c|c|}
\hline \multirow[t]{2}{*}{$\mathrm{NIPH}^{2}$} & & \multicolumn{2}{|c|}{ "Truth" } & \multirow[t]{2}{*}{$\Sigma$} \\
\hline & & - & + & \\
\hline \multirow{2}{*}{ PM } & - & 12 & 4 & 16 \\
\hline & + & 0 & 8 & 8 \\
\hline$\Sigma$ & & 12 & 12 & 24 \\
\hline \multicolumn{2}{|c|}{$\mathrm{NIBSC}^{\mathrm{b}}$} & \multicolumn{2}{|c|}{ "Truth" } & $\Sigma$ \\
\hline & & - & + & \\
\hline \multirow{2}{*}{ PM } & - & 4 & 2 & 6 \\
\hline & + & 8 & 10 & 18 \\
\hline$\Sigma$ & & 12 & 12 & 24 \\
\hline
\end{tabular}

asensitivity: $67 \%$; specificity: $100 \%$

bsensitivity: $83 \%$; specificity: $33 \%$ 